\title{
Psicoterapia cognitiva y religión: ¿Qué debe saber un terapeuta cognitivo de la diversidad religiosa de la Capital Federal y el Co- nurbano Bonaerense?
}

\section{Cognitive Psychotherapy and religion. What needs to know a cog- nitive therapist about religious diversity in Buenos Aires City and the Metropolitan Area?}

\author{
Guido Pablo Korman¹, Cristián J. Garay y Mercedes Sarudiansky \\ Facultad de Psicología, Universidad Nacional de Buenos Aires, y Centro Argentino de \\ Etnología Americana (CONICET), Argentina
}

(Recepción: Abril 2008 - Aceptación: Octubre 2008)

\begin{abstract}
Resumen
Los autores exploran la relación entre la psicoterapia y la religión. En primer lugar, examinan el fenómeno religioso en la práctica clínica. En segundo término, indaga el lugar de lo religioso en el Manual Diagnóstico y Estadístico de los Trastornos Mentales (DSM-IV). En tercer lugar, describe la relación entre desconocimiento y patologización de las creencias religiosas. En cuarto término, refiere el acercamiento entre la terapia cognitiva y la religión. En quinto lugar, describe la creciente importancia de la incorporación dentro de los modelos cognitivos de distintos sistemas de creencias a la práctica clínica y relaciona esta situación con el eclecticismo técnico propio de la terapia cognitiva. Por último, describe las distintas religiones existentes en la Ciudad Autónoma de Buenos Aires y el conurbano bonaerense a fin de manifestar la diversidad religiosa en nuestro medio.
\end{abstract}

Palabras claves: psicoterapia, religión, terapia cognitiva

\begin{abstract}
The authors explore the relationship between psychotherapy and religion. In first place, examine the religion in clinical practice. In second term, researches the place of the religion in the Diagnostic and Statistical Manual of Mental Disorders (DSM-IV). In third place, describes the relationship between ignorance and diagnosis of mental disorders based on religious beliefs. In fourth place, refers to the approach between cognitive therapy and religion. In fifth place, describes the incorporation of the beliefs systems to cognitive models in clinical practice and relates this situation to the technical eclecticism characteristically of the cognitive therapy. At last, describes the different religions that coexist in the Autonomous City of Buenos Aires and its suburbs, in order to show the religious diversity in this context.

Key words: psychotherapy, religion, cognitive therapy
\end{abstract}

1 Correspondencia: Guido Pablo Korman, E-mail: gkorman@psi.uba.ar 2) Cristian J. Garay, E-mail: cristiangaray@psi.uba. ar 3) Mercedes Sarudiansky, E-mail: msarudi@gmail.com 


\title{
Introducción
}

\begin{abstract}
"Una de las cosas que me gustan de verdad de la terapia cognitiva es que sirve de complemento a las creencias espirituales de cada uno. Sea cual sea su orientación religiosa, en el momento de la recuperación verá de pronto más hondo en sus propias raíces espirituales. Esto será así incluso si usted no es religioso y no cree en Dios. Todos tenemos valores y creencias que pueden ser fuente de opresión y ansiedad o de liberación y alegría."
\end{abstract}

\section{David Burns}

La psicología, la psiquiatría y la religión son descendientes de la rica historia del pensamiento humano. Estas disciplinas ofrecen una respuesta a la pregunta por el sentido de la vida, el sufrimiento y la enfermedad.

Desde el individuo como parte de un sistema cultural, la religión sirve como una lente para leer el mundo, el yo y las relaciones que se establecen entre ellos. La psicología, la psiquiatría y la religión se encuentran interesadas en cómo esta identidad es definida y afectada por procesos sociales y relaciones interpersonales.

El campo de la psicoterapia fue monopolizado, desde fines del siglo XIX hasta la década del 60, por el psicoanálisis y las corrientes derivadas del mismo. La impronta dejada por Freud dio lugar a un espacio de discrepancia entre la religión, la psicología y la psiquiatría (Nicholi, 2004).

Existe evidencia de que las tradiciones culturales determinan, en parte, la forma en que los individuos ven la salud mental y la física. Lo que es considerado saludable en una sociedad puede ser visto como no saludable en otra. Pese a ello, la mayoría de los profesionales del campo de la psicopatología pueden identificar a los trastornos psiquiátricos en casi todas las culturas; la forma y su expresión varían en muchas ocasiones y ello se encuentra generalmente relacionado con el sistema de creencias propio de cada cultura.

Los consumidores del sistema de salud combinan y utilizan distintas medicinas; por ejemplo, para resolver sus dolencias pueden recurrir a la utilización del yoga, a la vez que concurren al médico clínico o a una cura carismática (Idoyaga Molina, 2002). Esta conducta por parte de los usuarios del sistema de salud nos sitúa a los psicoterapeutas clínicos ante la necesidad de comprender los sistemas de creencias de nuestros pacientes.

Por ello podemos afirmar que la cultura, en el sentido amplio del término, es un elemento necesario al momento de valorar las cogniciones y diseñar la estrategia de intervención. El contexto cultural y las creencias que sostiene el paciente son esenciales para el psicoterapeuta de orientación cognitiva (Korman \& Garay, 2005). La cultura influye en todos los aspectos del proceso del tratamiento: la utilización de los tratamientos disponibles, las creencias en torno de la relación terapeuta-paciente y el valor de efectividad brindado a una determinada terapéutica (Keegan, 2007; Kirmayer, 2001).

Una de las creencias occidentales más comunes es la de la racionalidad, en donde las personas desviadas de ésta pueden ser consideradas enfermas. Esta asunción puede llevar a diagnósticos equivocados en personas de otras culturas, incluso subculturas, debido a que sus conductas pueden ser interpretadas como bizarras aisladas de su contexto. De hecho, las creencias y prácticas culturales de muchos grupos pueden ser vistas como patológicas; por ejemplo, nadie que haya visto al Diablo o a una bruja en forma de ave sería considerado enfermo dentro de la población campesina de San Juan, mientras que otras visiones en función del contexto denuncian la enfermedad. De la misma manera, ciertos tipos de religiosidad o espiritualidad pueden ser consideradas patológicas si ignoramos las creencias religiosas que subyacen (Lukoff, Lu \& Turner, 1992).

Las distintas religiones pueden ser consideradas como unidades cosmovisionales que impregnan la cultura. La tendencia a patologizar lo religioso y lo espiritual sin dar lugar al origen de donde surge la creencia o el grupo cultural del que emana, da cuenta de la falta de sensibilidad cultural. 
Así como en algunos lugares la educación en diversidad cultural se ha vuelto parte de programas del entrenamiento de psicólogos y psiquiatras, lo religioso y espiritual no se encuentran generalmente incorporados (Shafranske \& Malony, 1990).

\section{Lo religioso en la práctica clínica}

Las creencias y las prácticas religiosas pueden proveer soluciones a numerosos problemas de la vida; sin embargo, pueden a su vez crear conflicto, culpa y miedo (Patel, 1995).

Uno de los mitos de la comunidad psicológica y psiquiátrica es que las personas excesivamente religiosas presentan un riesgo mayor de psicopatología. El trabajo desarrollado por Hrmesh, MasserKavitzky y Gross-Isseroff (2003) en una comunidad de judíos ortodoxos es un indicador probable de que este juicio de la comunidad psicológica y psiquiátrica sea simplemente una arbitrariedad. Puede que este a priori se encuentre enmarcado en el etnocentrismo de la comunidad psicológica y psiquiátrica debido a que ésta es menos creyente que la población general. Bergin y Jensen en un trabajo realizado en EEUU encontraron que el 28\% de los clínicos psicólogos y el 21\% de los psiquiatras decían ser ateos o agnósticos, lo cual difería significativamente de la población general en la que el 6\% de la población declaraba ser atea o agnóstica (1990). En la Argentina no hay datos que hayan explorado la religiosidad de la comunidad psicológica y psiquiátrica.

\section{EI DSM-IV, lo Cultural y lo Religioso}

El Manual Diagnóstico y Estadístico de los Trastornos Mentales (DSM-IV) presenta una categoría específica en cuanto a los fenómenos religiosos dentro del Eje I: “Otros problemas que pueden ser objeto de atención clínica: Problema religioso o espiritual” (APA, 1995). En el desarrollo del DSM el lugar de la cultura fue variando significativamente. El DSM-III no hace referencia a la cultura, el DSM-IIIR (revisado) hace sólo una referencia breve a la cultura (Rogler, 1993), la cual se hace presente con fuerza recién en el DSM-IV. Éste incluye información en la cual prescindir del contexto cultural puede llevar a diagnósticos errados (O’Connor \& Vanderberg, 2005). Pese a este reciente cambio en la conceptualización del rol de la cultura a la hora de evaluar los comportamientos y las conductas, existe una fuerte controversia respecto de este rol que aún no ha sido resuelta. Algunos de los autores que confeccionaron la parte cultural del DSM-IV fueron muy críticos respecto al lugar otorgado a la cultura en el manual. La mayor parte de los argumentos recayó en que la fuerte adherencia al modelo biológico deja de lado la influencia de lo social en la enfermedad mental (Good, 1996). Otros autores argumentan que la cultura configura la experiencia de malestar de manera tal que debe ser considerada parte sustancial de las decisiones diagnósticas y que no puede ser reducida a una nota al pie dentro de la consideración biológica (Alarcón, 1995; Lewis-Fernández, 1996). Otra área importante en la que el DSM-IV hace referencia al rol de la religiosidad es en la definición de idea delirante.

"Idea delirante: Falsa creencia basada en una inferencia incorrecta relativa a la realidad que es firmemente sostenida, a pesar de lo que casi todo el mundo cree y a pesar de cuanto constituye una prueba o evidencia incontrovertible y obvia de lo contrario. La creencia no está aceptada ordinariamente por otros miembros de la subcultura o cultura a la que el sujeto pertenece (p. ej., no es un artículo de fe religiosa)...” (p. 783). Mediante esta definición el DSM-IV, implícitamente, da a la religión el lugar de una cultura o subcultura, consideración que eximiría las creencias religiosas de significación patológica (O’Connor \& Vanderberg, 2005). Si una creencia se encuentra relacionada con un tipo particular de religión, ella no debería poseer significación patológica al momento de encontrarse compartida por otras personas.

\section{Desconocimiento y patologización}

Desde la perspectiva de los profesionales de la salud mental, muchas prácticas religiosas pueden ser consideradas patológicas, si no es tenido en cuenta el contexto. Es interesante mencionar el trabajo realizado por O’Connor y Vanderberg (2005). Dichos autores investigan la valoración que profesionales de la salud mental hacen de la significación patológica de algunas creencias religiosas. 
El mencionado estudio expone tres religiones presentes en EEUU: Catolicismo, Mormones y La Nación del Islam. El catolicismo es una religión con muchos seguidores en el país de los autores. Las otras dos religiones, los Mormones y La Nación del Islam, poseen menos seguidores, pero como hecho a destacar ambas surgen en los EEUU, a la vez que ambas religiones presentan creencias bastante distintas de las católicas. Las hipótesis con las que estos autores trabajan son las siguientes: En primer lugar, consideran que los clínicos estarán más familiarizados con las creencias del Cristianismo, mientras que las creencias de los mormones puede que les resulten menos familiares, y las de la Nación del Islam menos familiares aún. De esto se presupondrá que las creencias serán consideradas menos patológicas en la medida en que éstos tengan mayor conocimiento de las mismas y que, en esta medida, actuarán conforme a las recomendaciones del DSM-IV. En segundo lugar, hipotetizan que los rangos de patología serán más pronunciados en las religiones menos conocidas.

La tercera hipótesis es que las consecuencias de esas creencias serán más patologizadas a partir de la posibilidad de que generen daños a otros.

Todos los participantes (110 profesionales de la salud mental) habían tenido entrenamiento en el Diagnóstico con DSM-IV.

Cada participante recibió información concerniente a la edad, el sexo, la filiación religiosa y el grado de estudio obtenido. Se presenta al clínico una serie de viñetas en las que el consultante ha adoptado una serie de nuevas creencias entre 6 y 12 meses atrás. Las creencias adoptadas por estos individuos ficticios responden a las tres religiones elegidas para este estudio. Para las creencias Católicas fueron elegidas las pertenecientes a la intervención del Espíritu Santo, transubstanciación, pecados particulares y las ideas sobre la otra vida. Para los mormones fueron identificadas creencias acerca de la naturaleza de Dios, el origen del alma, las restricciones dietarias y la existencia de otra vida. Para la Nación del Islam, se incluyeron creencias respecto la intervención de Alá, los orígenes de los distintos grupos de personas, algunas restricciones dietarias y el origen del demonio.

Algunos de los ejemplos que utilizan para las distintas viñetas merecen ser mencionados. "Franco se vuelve Católico en el último año y cree que el Espíritu Santo le dio una fuerza especial para defender la fe". "Juan cree apasionadamente en los mandatos de los Mormones y, por ende, que luego de su muerte será transformado en dios". De manera similar "William cree en la revelación de que una nave espacial sobrevuela EEUU desde 1929” (O’Connor \& Vanderberg, 2005).

Cada participante recibe una serie de seis viñetas. Tres viñetas de distracción y tres viñetas correspondientes a los cuatro grupos armados (religión identificada, pero sin peligro para otros; religión identificada, pero con peligro para otros; religión no identificada, pero sin peligro para otros y religión no identificada, pero con peligro para otros).

La principal hipótesis que sostenían los autores es que cuanto más conocida era la religión, ésta sería considerada menos patológica. La hipótesis resultó confirmada: los indicadores de patología fueron más altos para la Nación del Islam; en segundo lugar se ubicaron los mormones y, por último, los católicos.

La segunda hipótesis del estudio también resultó confirmada. Las creencias que no se hallaban explícitamente identificadas con una religión resultaron ser consideradas más patológicas. Al mismo tiempo, identificar la religión implicó considerar menos patológica la creencia que se sostenía en la viñeta, siempre y cuando fuera la religión más conocida (católica); en la medida en que la religión es menos conocida, se incrementaba la patología.

Una tercera hipótesis que se corroboró es que cuanto más asociada a un daño a terceros se encontraba la creencia, ésta habría de ser más patológica, al igual que en las otras hipótesis: cuanto menos conocida es la religión, más patológica es la evaluación de los clínicos respecto a esta circunstancia.

La investigación arroja como resultados que cada creencia es evaluada de manera distinta por parte de los clínicos y ello no guarda relación con su racionalidad (ninguna de las tres presentan ideas relacionadas con la racionalidad, sino con las creencias propias de cada una de las religiones 
tomadas para la investigación). Pese a las recomendaciones propuestas por el DSM-IV, los clínicos han considerado más patológicas las creencias de la Nación del Islam aun cuando en la viñeta fuera consignada la pertenencia a tal religión. Esta información se vuelve relevante cuando se ve confirmada que la pertenencia al Catolicismo o a los Mormones implicaba menor nivel de patología. Las consecuencias del desconocimiento por parte de los profesionales, y consecuentemente el diagnóstico errado, daba lugar al diagnóstico de los más serios trastornos mentales. El rol de la cultura en la génesis de las conductas y las creencias debe tener una mayor consideración en el DSM-IV, de manera que se puedan evaluar mejor (O’Connor \& Vanderberg, 2005).

\section{Acercamiento entre la Terapia Cognitiva-Conductual y la religión}

En una reciente publicación Anderson y Asmundson (2006) explicitan la importancia de tomar en cuenta la relación entre terapia cognitiva-conductual (TCC) y religión.

Esta relación descansa principalmente en el hecho de que la TCC presenta un modelo de trabajo que se apoya sobre el respeto de las creencias de los pacientes. Esto es posible debido a que ellas inciden en la forma en que se lee el mundo. Las personas sostienen creencias desde las que evalúan su desempeño en la vida. Según esta corriente teórica, las creencias no deben ser evaluadas en términos de "buenas" o “malas", sino leídas desde su funcionalidad.

Como ejemplo de ello, podemos ubicar el caso de aquellas personas que ligan sus creencias a una divinidad. En el caso de que crean que hay un conjunto de normas dadas por Dios (o alguna divinidad) significarán, evaluarán e intentarán obrar acorde a ellas. No todas las personas que creen en un orden ligado a la divinidad desarrollan trastornos mentales; ello permite discutir la cosmovisión del paciente en términos operacionales en los que las creencias religiosas son parte de la discusión. Esta discusión se lleva a cabo desde la propia lógica interna de las creencias del paciente.

\section{Terapia cognitiva e incorporación de distintos sistemas de creencias}

Dentro de la terapia cognitiva, existe una vasta literatura que da cuenta de los intentos de incorporar herramientas de otras disciplinas a la práctica psicoterapéutica; esta complementariedad ha dado lugar a lo que son los llamados desarrollos de psicoterapia cognitiva de tercera generación (Anderson \& Asmundson, 2006). Estos modelos llevan a incorporar elementos propios de los distintos sistemas religiosos, tal como se advierte en el número importante de clínicos y teóricos que han escrito extensamente acerca de la integración del budismo y los principios de la terapia cognitiva. El foco de estas discusiones está centrado principalmente en la importancia del control del pensamiento, tal como lo demuestra el tratamiento para el trastorno límite de la personalidad (TLP) desarrollado por Marsha Lineham (1993), quien toma herramientas de su aprendizaje como monje budista para desarrollar el modelo actualmente más exitoso en el tratamiento del TLP.

Teasdale, Segal y Williams proponen la integración de meditación, conciencia plena y terapia cognitiva para reducir los riesgos de recaídas y recurrencias en la depresión (Teasdale et al., 2000; Lau, 2005), idea ya implementada en la reducción del estrés por parte de Kabat-Zinn (1990).

Por otra parte, Williams (Williams et al., 2006) propone la utilización de métodos de meditación y conciencia plena integrados con la terapia cognitiva para evitar la reactivación de la ideación suicida. Este método se basa en la idea de que los participantes desarrollan habilidades de aceptación y evitación de juicios de valor en lo que hace a la ideación suicida.

Debemos mencionar además que la utilización de recursos religiosos no es privativa de la terapia cognitiva sino que es un fenómeno evidente en distintas líneas teóricas. Es así como en el psicoanálisis diversos autores han propuesto integrar la práctica de la mente plena (mindfulness) como una forma de enriquecer el funcionamiento de esta terapia (Rubin, 1996).

Elámbito de la complementariedad ha ampliado no sólo el nivel técnico sino también la perspectiva del especialista respecto de la utilización de las creencias propias de los pacientes, como lo muestran los desarrollos de Richards y Bergin (1997). Estos autores proveen un amplio fundamento para la integración de herramientas espirituales en la práctica de la psicoterapia. Asimismo, Boehnlein 
(2005) sugiere el aumento de desarrollos psicoterapéuticos que enfatizan en la implementación de instancias espirituales.

La cuestión central en aquellos que llevan a cabo investigaciones acerca de las intervenciones psicoterapéuticas es: ¿Qué tratamiento funciona para quién y en qué condiciones? Enmarcada dentro de esta misma lógica, Propst, como terapeuta de orientación cognitiva (1992), compara la eficacia de la terapia cognitiva religiosa y no religiosa para el tratamiento de la depresión en pacientes religiosos. Según esta autora, la terapia cognitiva presenta ciertos valores, tales como autonomía y eficacia personal, que son contrarios a los valores religiosos, en los cuales la vida guarda dependencia con la divinidad (Propst et al., 1992). Esta discrepancia de valores llevaría a una subutilización de los servicios de salud mental por individuos muy religiosos, problema que podría ser subsanado gracias al eclecticismo técnico de la TCC. A continuación consignaremos un caso donde se estudian distintos tipos de intervenciones.

En este estudio, los autores aplicaron dos formas distintas de tratamiento en pacientes religiosos. Un grupo de pacientes recibió terapia cognitivo-conductual "religiosa" y en otro grupo la misma cantidad de pacientes recibieron el tratamiento cognitivo usual. La muestra incluía un total de cincuenta y nueve pacientes que cumplían criterios diagnósticos para un trastorno depresivo mayor sin síntomas psicóticos.

El tratamiento tuvo una duración estándar de entre dieciocho y veinte sesiones. Intervinieron terapeutas religiosos y no religiosos en ambos grupos. Se trabajó con dos grupos control de aproximadamente diez a once pacientes por grupo. El primer grupo control recibió orientación pastoral (pastoral counselling) y el otro se conformó con los pacientes en lista de espera. El seguimiento se realizó durante dos años. Los resultados indicaron que la terapia cognitiva con contenido religioso y la consulta pastoral mostraron mejores resultados que la terapia cognitiva sin contenido religioso y la lista de espera. Esta investigación resalta la importancia de considerar adaptaciones de los tratamientos psicológicos estándar para aumentar -o preservar- su eficacia en pacientes con valores religiosos específicos. Los resultados favorables alcanzados por la consulta pastoral y la terapia cognitiva religiosa sugieren que la visión del mundo del paciente puede tener un impacto mayor que los ingredientes activos de un tratamiento que entre en colisión con ellos. Otro dato interesante de este estudio es que dentro de los tratamientos psicológicos, el grupo que obtuvo los mejores resultados fue el de los terapeutas no religiosos que aplicaban el tratamiento cognitivo con orientación religiosa. Este dato empírico es importante porque va en contra de la suposición generalizada de que un terapeuta que comparta los valores religiosos del paciente tendrá más eficacia que uno que no los comparta. El estudio sugiere que lo más importante es el conjunto de valores que va implícito en los procedimientos y experiencias utilizados para lograr el cambio terapéutico, así como la capacidad del terapeuta de respetar esos valores, aun cuando no los comparta.

Otras formulaciones que siguen esta lógica son propuestas por D’Souza y Rodrigo (2004), quienes sugieren la incorporación de lo espiritual para aumentar los efectos de la terapia cognitiva, proponiendo en el transcurso del tratamiento el uso de la meditación y la validación e incorporación del propio sistema de creencias, puesto que ello resulta beneficioso para el paciente. Los mismos autores entienden que esta modificación del tratamiento permite integrar a toda "la persona" de una manera holista y no parcializada.

Se podría decir entonces que algunos de los últimos desarrollos teóricos en el campo de la psicoterapia de orientación cognitiva focalizan en la incorporación de los propios sistemas de creencias de los clientes debido a que inciden en la atención de la salud mental. Por otra parte, ciertas herramientas que eran propias de los tratamientos alternativos están siendo incorporadas en el desarrollo de nuevos tratamientos de orientación cognitiva, en función de que son investigados en combinación con terapia cognitiva; de obtener buenos resultados, empiezan a formar parte de las herramientas de los psicoterapeutas, como es el caso de las técnicas de meditación. 


\section{Eclecticismo técnico de la TC}

El modelo de la terapia cognitiva se centra en el papel de los procesos cognitivos, esto es, en el modo que se significan e interpretan las experiencias a lo largo de la vida. Se considera que dichos procesos tienen un rol fundamental en la aparición y el mantenimiento de los trastornos y problemas que aquejan al ser humano.

La terapia cognitiva presenta un eclecticismo técnico (Alford y Norcross, 1991 citado por Fernández-Álvarez, 1992; Clark y Beck, 1997), lo que permite una gran variabilidad en las técnicas de intervención. Este hecho se encuentra respaldado por la gran cantidad de enfoques cognitivos que presentan diversas técnicas de intervención en función del trastorno específico.

Debido al eclecticismo técnico, la terapia cognitiva da lugar a la complementariedad terapéutica (utilizar distintas estrategias terapéuticas en función del paciente). En la actualidad, la terapia cognitiva no es homogénea (Beck, 2005). Hay distintas líneas teóricas que proponen sus propias ideas en torno al hombre saludable; ello implica una serie de posibilidades de complementariedad que permiten la diversidad de tratamientos y la posibilidad de adaptarlos a la perspectiva del cliente. Sirva de ejemplo la recomendación del yoga como estrategia complementaria hecha por los psicoterapeutas cognitivos en relación con muchos de los pacientes que se hallan englobados en los trastornos de ansiedad en la Ciudad de Buenos Aires (Korman y Garay, 2006). Este fenómeno da cuenta de la utilización por parte de terapeutas cognitivos del yoga a partir de una lectura particular del mismo, despojado mayoritariamente del contenido teórico de la práctica del yoga (Korman y Saizar, 2006; y Korman, Saizar y Garay, 2006).

\section{Los religiosos no son parte representativa en las muestras en la población de los EEUU, ¿qué ocurre en la Capital Federal y el Conurbano Bonaerense en Argentina?}

Algunos autores postulan que los religiosos no son representativos en las muestras de las investigaciones psiquiátricas (Larson et al., 1986; Larson et al., 1989). Varios factores pueden estar involucrados en estefenómeno. Entre ellos, el estigma quela consultapsiquiátrico-psicológica conlleva en muchas comunidades, para quienes la realizan. Es frecuente que las personas que llevan a cabo una consulta con un profesional de la salud mental refieran sentimientos de vergüenza, incomodidad y soliciten al profesional discreción. Asimismo, muchas personas consideran que los tratamientos farmacológicos están asociados a cuadros psiquiátricos mayores (esquizofrenia, psicosis, etc.), creencia que convierte a este recurso terapéutico en una estrategia subutilizada por tal población. A su vez, podemos inferir que hay distintos factores involucrados en este fenómeno. Uno de ellos es el desconocimiento de los sistemas de creencias que los pacientes poseen por parte de los profesionales de la salud mental, lo que genera un gran temor a ser rechazados y evaluados negativamente por sus creencias. Considerando que en la Argentina hay una gran diversidad de creencias religiosas, nos podemos preguntar: ¿Los profesionales de la salud mental conocen las diversas religiones de la población que habita en la zona mencionada? ¿Conocen las distintas cosmovisiones del mundo que las diversas religiones poseen y sus evaluaciones respecto del padecimiento mental? Aún no tenemos datos para responder esta pregunta. Sin embargo, creo necesario mencionar las distintas religiones que se presentan en Capital Federal y el Conurbano Bonaerense para que los profesionales del campo de la salud mental den cuenta de esta diversidad religiosa. Los datos para esta descripción han sido tomados de la Guía de la Diversidad Religiosa de Buenos Aires (Forni, Mallimaci y Cárdenas, 2003): Afrobrasileños, Budistas (Jodo Shinshu, Soka Gakkai, Ha Ma-Um; Asociación Budista China en la Argentina y Kagyu Tekchen Chöling), Católicos Apostólicos Romanos (Catolicismo Integral, Catolicismo de la renovación carismática y Catolicismo de la pluralidad), Católicos Ortodoxos (Iglesia Ortodoxa Griega, Iglesia Apostólica Rusa en el Extranjero, Iglesia Apostólica Armenia e Iglesia Católica Apostólica Ortodoxa de Antioquía), Católicos Protestantes (Iglesia Evangélica Metodista Argentina, Iglesia Evangélica del Río de la Plata, Iglesia Evangélica Luterana Argentina, Iglesia Evangélica Luterana Unida, Iglesia Evangélica Luterana Dano-Argentina, Iglesia Anglicana de la Argentina, Iglesia Evangélica Presbitereana de San Andrés, Iglesia Evangélica Menonita, Iglesia Cristiana Evangélicas, Iglesia Evangélica Bautista, Alianza Cristiana y Misionera, Ejército de Salvación, Iglesia Evangélica Congregacional Armenia Santísima Trinidad, Junta Americana de 
Misiones a Israel, Iglesia Evangélica del Nazareno, Iglesia Nueva Apostólica e Iglesia Adventista del Séptimo Día), Protestantes Pentecostales (Iglesia Cristiana Bíblica, Asociación Evangélica Asamblea de Dios, Unión de Asambleas de Dios, Movimiento Cristiano y Misionero, Asociación la Iglesia de Dios e Iglesia Visión de Futuro), Posprotestantes (Iglesia de Jesucristo de los Santos de los Últimos Días y Testigos de Jehová), Espiritistas (Confederación Espiritista Argentina, Escuela Científica San Basilio y Casa de Oración y Beneficiencia del Culto Cristiano), Hinduistas (Sociedad Internacional para la Conciencia de Krishna y Organización Sri Sathya Sai Baba Mundial), Islámicos (sunnita y shiíta), Judíos (Jabad Lubavitch; Yesoth Hadth y Congregación Emanu-El) y Teosofías y centros de estudio (Sociedad Teosófica, The Summit Lightouse, Fundación Hastinapura, Sociedad Antroposófica, Asociación Cultural Nueva Acrópolis y Movimiento Gnóstico Cristiano Universal de la Argentina).

Luego de nombrar el gran número de alternativas religiosas presentes en nuestro medio, volvemos a preguntarnos: ¿los psicoterapeutas conocen esta diversidad? Este es el desafío de la continuidad de nuestra investigación.

\section{Palabras finales}

Las terapias cognitivas son tratamientos psicológicos que utilizan una gran variedad de recursos que actúan indistintamente sobre la esfera de la emoción, de la cognición y de la conducta. En la actualidad, las psicoterapias de orientación cognitiva han sumado su interés en áreas distintas, que incluyen los procesos metacognitivos, las emociones, técnicas de conciencia plena, entre otras herramientas, y la incorporación de los sistemas de creencias en sus tratamientos.

La relación entre el tratamiento psicoterapéutico y las creencias religiosas es un área que se encuentra en desarrollo. Hay pocos tratamientos específicos que tomen como modelo lo religioso y el trastorno mental dentro de la terapia cognitiva.

En este marco, los terapeutas de orientación cognitiva dentro del contexto local estamos lejos de desarrollar tratamientos específicos para nuestra población. Sin embargo, creemos necesario que los psicoterapeutas de dicha orientación conozcan los sistemas de creencias de sus pacientes e intenten respetar las consignas propuestas por el DSM-IV de tomar a la cultura como un elemento significativo a la hora de realizar el diagnóstico. Es decir, los efectores de los servicios de salud deben entender a sus pacientes y no los usuarios del sistema de salud cambiar sus sistemas de creencias para poder utilizar los servicios de este sistema. Por ello, es esencial que los psicoterapeutas sepan de la existencia de las distintas cosmovisiones religiosas debido a que ellas implican sistemas de creencias específicos que operan en la cosmovisión del mundo de las personas a las que asisten. Conocer estas creencias nos ayudará, en primer lugar, a no patologizar una experiencia religiosa y, en segundo término, a comprender mejor la cosmovisión del paciente que consulta, generando una mayor comprensión entre psicoterapeuta y consultante.

\section{Referencias}

Alarcón, R.D. (1995). Culture and psychiatric diagnosis: Impact on DSM-IV and ICD-10. Psychiatric Clinics of North America, 18, 449-465.

Anderson, G., Asmundson, G.J.G. (2006). Cognitive Behaviour Therapy, 35 (1), 1-2.

American Psychiatric Association (1995). Manual diagnóstico y estadístico de los trastornos mentales. Barcelona: Masson.

Beck, A.T. (2005). The Current State of Cognitive Therapy. A 40-Year Retrospective. Archives of General Psychiatry, 62: 953-959.

Bergin, A.E., Jensen, J.P. (1990). Religiosity of psychotherapists: A national survey. Psychotherapy, 27: 3-7.

Boehnlein, J.K. (2005). The Convergence of Mind and Spirit. Washington and London: American 
Psyquiatric Press.

Burns, D. (2006). Adiós, ansiedad. Cómo superar la timidez, los miedos, las fobias y las situaciones de pánico. Buenos Aires: Paidós.

Clark, D.M., Beck, A.T. (1997). El estado de la cuestión en la teoría y la terapia cognitiva. En Caro I. (comp.): Manual de psicoterapias cognitivas. Barcelona: Paidós.

D’Souza, R.F., Rodrigo, A. (2004). Spiritually augmented cognitive behavioural therapy. Australas Psyquiatry, 12 (2), 148-52.

Fernández-Álvarez, H. (1992). Fundamento de un modelo integrativo en psicoterapia. Buenos Aires: Paidós.

Forni, F., Mallimaci, F., Cárdenas, L. A. (2003). Guía de la diversidad religiosa de Buenos Aires. Buenos Aires: Biblos.

Good, B.J. (1996). Culture and DSM-IV: Diagnosis, knowledge and power. Culture, Medicine \& Psyquiatry, 20, 127-132.

Hermesh, H., Masser-Kavitzky, R., Gross-Isseroff, R. (2003). Obsessive-compulsive disorder and Jewish religiosity. Journal of Nervous and Mental Disease, 191, 201-203.

Idoyaga Molina, A. (2002). Culturas, enfermedades y medicinas. Reflexiones sobre la atención de la salud en contextos interculturales de Argentina. Buenos Aires: CAEA-CONICET.

Kabat-Zinn, J. (1990). Full Catastrophe Living: Using the Wisdom of your mind to Face Stress, Pain and Illness. New York: Dell Publishing.

Keegan, E. (2007). Ensayos de Terapia Cognitiva. Buenos Aires: EUDEBA.

Kirmayer, L.J. (2001). Cultural Variations in the Clinical Presentation of Depression and Anxiety Implications for Diagnosis and Treatment. Journal of Clinical Psyquiatry, 62 (suppl 13), 22-28.

Korman, G. (2006). Depresión y Taxa Vernáculos en la Atención de la salud en contextos interculturales. Tesis Doctoral IUNA-CAEA-CONICET. Inédito.

Korman, G. y Garay, C. (2005). Prácticas terapéuticas con alusión a lo sagrado y su relevancia para el psicólogo clínico de orientación cognitiva. Revista Argentina de Clínica Psicológica, 13 (3), 45-51.

Korman, G. y Saizar, M. (2006). Reflexiones en torno a la inclusión del yoga como terapia complementaria en los tratamientos psicoterapeúticos cognitivos en Buenos Aires. Revista Brasileira de Sociologia da Emoção, 5 (13), 97-110.

Korman, G., Garay, C. y Saizar, M. (2006). La recomendación por parte de psicoterapeutas cognitivos de la práctica del yoga como estrategia complementaria de la terapia. Memorias de las XIII Jornadas de Investigación de la Facultad de Psicología / UBA, Tomo III, 433-435.

Larson, D., Donahue, M., Lyons, J., Benson, P., Worthington, E., Blazer, D. (1989). Religious affiliations in mental health research samples as compared with national samples. Journal of Nervous and mental disease, 177, 109-111.

Larson, D., Pattison, M., Blazer, D., Omran, A. \& Kaplan, B. (1986). Systematic analysis of research on religious variables in four major psyquiatric journals, 1978-1982. American Journal of Psychiatry, 143, 329-334.

Lau, M.A. (2005). Terapia Cognitiva basada en la Conciencia Plena: Integrando la Conciencia plena, la meditación y la terapia cognitiva para reducir el riesgo de la recaída en la depresión. Revista Argentina de Clínica Psicológica, 13 (3): 7-15.

Lewis-Fernández, R. Cultural formulation of psychiatric diagnosis. (1996). Culture, Medicine \& Psyquiatry, 20, 133-144. 
Lukoff, D., Lu, F. \& Turner, R. (1992). Toward a more culturally sensitive DSM-IV: Psychoreligious and psychospiritual problems. Journal of Nervous and Mental Disease, 180, 673-682.

Lineham, M. (1993). Cognitive-Behavioral Treatment of Borderline Personality Disorder. New York: Guilford Press.

Nicholi, A.M. (2004). Introduction: Definition and Significance of a Worldview. En Josephson, A.M., Peteer, J. R. (comp.): Handbook of Spirituality and Worldview in clinical practice. Arlington: American Psychiatric Publishing, Inc.

O’Connor, S., Vanderberg, B. (2005). Psychosis or Faith? Clinician's Assessment of Religious Belief. Journal of Consulting and Clinical Psychology, 73 (4), 610-616.

Patel, V. (1995). Spiritual distress: an indigenous model of nonpsychotic mental illness in primary care in Harare, Zimbabwe. Acta Psychiatrica Scandinava, 92: 103-107.

Propst, L.R., Ostrom, R., Watkns, P., Dean, T., Mashburn, D. (1992). Comparative efficacy of religious and nonreligious cognitive-behavioral therapy for the treatment of clinical depression in religious individuals. Journal of Consulting and Clinical Psychology, 60 (1), 94-103.

Richards, P., Bergin, A.E. (1997). Handbook of psychotherapy and religious diversity. Washington: American Psychological Association.

Rogler, L.H. (1993). Culture in psychiatric diagnosis: An issue of scientific accuracy. Psychiatry, 56, 324-327.

Rubin, J.B. (1996). Psychotherapy and Buddhism. New York: Plenum.

Shafranske, E.P., Malony, H.N. (1990). Clinical psychologists' religious and spiritual orientations and their practice of psychotherapy. Psychotherapy, 27, 72-78.

Teasdale, J.D., Segal, Z.V., Ridgeway, V.A., Soulsby, J. M. y Lau M. A. (2000). Prevention of relapse/ recurrence in major depression by mindfulness-based cognitive therapy, Journal of Consulting and Clinical Psychology, 68: 615-623.

Williams, J.M., Duggan, D.S.; Crane, C.; Fennell, M.J. (2006). Mindfulness-Based cognitive therapy for prevention of recurrence of suicidal behavior. Journal of Consulting and Clinical Psychology, 62, 201-210. 